\title{
Reduced T Cell Receptor Excision Circle Levels in the Colonic Mucosa of Microscopic Colitis Patients Indicate Local Proliferation rather than Homing of Peripheral Lymphocytes to the Inflamed Mucosa
}

\author{
Ashok Kumar Kumawat, ${ }^{1}$ Kristina Elgbratt, ${ }^{1}$ Curt Tysk, ${ }^{1,2}$ \\ Johan Bohr, ${ }^{1,2}$ and Elisabeth Hultgren Hörnquist ${ }^{1,3}$ \\ ${ }^{1}$ School of Health and Medical Sciences, Örebro University, 70182 Örebro, Sweden \\ ${ }^{2}$ Division of Gastroenterology, Department of Medicine, Örebro University Hospital, 70185 Örebro, Sweden \\ ${ }^{3}$ School of Medicine, Örebro University, 70182 Örebro, Sweden
}

Correspondence should be addressed to Ashok Kumar Kumawat; ashok.kumawat@oru.se

Received 23 April 2013; Revised 20 June 2013; Accepted 24 June 2013

Academic Editor: Tadayuki Oshima

Copyright (C) 2013 Ashok Kumar Kumawat et al. This is an open access article distributed under the Creative Commons Attribution License, which permits unrestricted use, distribution, and reproduction in any medium, provided the original work is properly cited.

\begin{abstract}
Dysregulated $\mathrm{T}$ cell responses in the intestine may lead to chronic bowel inflammation such as collagenous colitis (CC) and lymphocytic colitis (LC), together known as microscopic colitis (MC). Having demonstrated increased local T cell responses in the intestinal mucosa of MC patients, we investigated the recent thymic emigrants by measuring $\mathrm{T}$ cell receptor excision circle (TREC) levels in the colonic biopsies from CC $(n=8)$, LC $(n=5)$, and CC or LC patients in histopathological remission (CC-HR, $n=3$ ) (LC-HR, $n=6)$, non-inflamed diarrhoea patients $(n=17)$, and controls $(n=10)$ by real-time PCR. We observed lower median TREC levels in both CC and LC patients as well as in LC-HR patients compared to controls. In contrast to MC patients, non-inflamed diarrhoea patients presented with enhanced TREC levels compared to controls. None of the recorded differences did, however, reach statistical significance. A trend towards increased relative expression of CD3 was noted in all MC subgroups examined and reached statistical significance in LC patients compared to controls. In conclusion, reduced TRECs level in the colonic mucosa, together with our previously demonstrated enhanced expression of Ki67 ${ }^{+} \mathrm{T}$ cells, suggests local expansion of resident $\mathrm{T}$ lymphocytes in the inflamed mucosa of MC patients.
\end{abstract}

\section{Introduction}

Microscopic colitis (MC) is a chronic inflammatory bowel condition of unknown aetiology comprising collagenous colitis (CC) and lymphocytic colitis (LC) with an annual incidence each of 5-6 cases per 100.000 individuals [1-3]. Although the data on pathophysiology are still limited, it is postulated that $\mathrm{MC}$ is caused by disturbed immune responses to luminal antigen(s) in predisposed individuals [4].

It is generally appreciated that aberrant $\mathrm{T}$ cell responses may lead to chronic gut inflammatory conditions like inflammatory bowel disease [5]. Careful evaluation of thymic activity is of great interest since new naive $\mathrm{T}$ cells are made immunocompetent in thymus. Thymectomy has previously been demonstrated to prevent relapse in ulcerative colitis (UC) patients [6]. Our previous studies on $\mathrm{G} \alpha \mathrm{i} 2^{-/-}$spontaneous and dextran-sodium-sulphate- (DSS-) induced mouse models of colitis demonstrated aberrant thymocyte development characterized by enhanced numbers of mature and decreased number of immature thymocytes before and during onset of colitis, as well as decreased migratory responsiveness to intrathymic chemokines $[7,8]$.

The $\mathrm{T}$ cell receptor (TCR) consists of either of two types of heterodimers: $\alpha \beta$ TCRs or $\gamma \delta$ TCRs. Generation of T cell repertoires with diverse antigen specificities is achieved by random rearrangement of TCR gene segments (V-D$\mathrm{J}$, variable, diversity, and joining) in thymus $[9,10]$. This process is initiated by recognition of recombination signal 
sequences (RSS) that flank the coding sequence and during this process the two signal ends are circularized, forming an extrachromosomal circular excision product [10]. These socalled T cell receptor excision circles-TRECs-are stable but are not replicated during mitosis and are consequently diluted with each cell division $[11,12]$. Thus, TRECs levels are a direct reflection of the amount of recent thymic emigrants (RTE) in the periphery. TRECs measurements are used extensively to document $\mathrm{T}$ cell reconstitution following treatment of HIV infection and in patients who have undergone haematopoietic stem cell transplantation $[9,13,14]$.

Increased mucosal mRNA expression of IFN- $\gamma$ and TNF$\alpha$ has been observed in the colonic mucosa of both CC and LC patients [15]. Recent flow cytometric analyses by our group have shown heavy infiltration of $\mathrm{CD} 8^{+}$intraepithelial lymphocytes (IELs) in the mucosa of CC and especially in LC patients [16]. Whether the heavy infiltration of $\mathrm{CD}^{+}$IELs is due to a larger influx of T cells, recent thymic emigrants, or an increased expansion of resident $\mathrm{T}$ cells in the mucosa of CC and LC patients, is still unknown. Such information is a first step toward understanding whether the activating antigen(s) resides in the mucosa or is rather transported via, for example, dendritic cells to the draining lymph nodes to activate naïve $\mathrm{T}$ cells there, thereby adding information on the nature of the chronic colonic inflammation in the mucosa of MC patients.

The aim of this study were to investigate the TREC levels in the $\mathrm{CD}^{+} \mathrm{T}$ cell compartment in the colonic mucosa of CC and LC patients compared to controls, using real-time PCR analysis for CD3 mRNA expression and real-time PCR analysis for TRECs analysis.

\section{Material and Methods}

2.1. Patients. Clinically active LC and CC were defined as $\geq 3$ loose or watery stools/day and/or abdominal pain. The diagnostic criteria for LC were histological findings (Figure 1(b)) of increased numbers of IELs ( $\geq 20 / 100$ surface epithelial cells) in conjunction with surface epithelial cell damage and infiltration of lymphocytes in the lamina propria, but a normal collagen layer [3]. In CC, in addition to lymphocytic infiltration in the lamina propria and the epithelium, deposition of a subepithelial collagen layer of $\geq 10 \mu \mathrm{m}$ is seen (Figure 1(c)) [3].

MC patients fulfilling the above histopathological criteria and previously diagnosed with CC, LC, or UC and ongoing clinically active disease were recruited in the study. Exclusion criteria for both MC patients and diarrhoea controls were a previous history of Crohn's disease and/or clinical signs of gastrointestinal infection, ischemic colitis, or neoplastic disease.

Colon biopsy specimens from 8 patients with CC, 5 with LC, and 4 with UC were investigated. In addition, we also identified a subgroup of MC patients who were previously diagnosed with LC $(n=6)$ or CC $(n=3)$, but who at the time of biopsy collection showed no histological signs of inflammation despite clinical symptoms of active LC or CC. Biopsies from these patients were grouped separately and are referred to as LC-histopathological remission (LC-HR) and CC-histopathological remission (CC-HR). The colonoscopic examination revealed that $\mathrm{CC}$ and LC patients generally had normal mucosa but occasionally with slight oedema or erythema. We also investigated biopsies from 17 patients with chronic, nonbloody diarrhoea, but with histologically normal mucosa and no earlier diagnosis of MC or IBD $(n=$ 17) who are referred to as noninflamed diarrhoea patients. The noninflamed diarrhoea patients had an endoscopically normal mucosa, except for insignificant findings such as diverticulosis or polyps in some patients. Finally, 10 controls without diarrhoeal symptoms were recruited among patients undergoing colonoscopy for examination of gastrointestinal bleeding or abnormal radiological findings. All had normal findings at colonoscopy and histopathologic examination of colonic biopsies. None of the patients, irrespective of being in histological remission or having active disease, were treated with immunosuppressive drugs or antibiotics. The demographic features of the patients included in the study, as well as information on their use of nonsteroidal antiinflammatory drugs (NSAIDs) and proton pump inhibitors (PPIs), are presented in Table 1.

Six biopsies from each individual were obtained using standard biopsy forceps, placed immediately in RNAlater (Ambion, Austin, TX, USA), and stored at $-80^{\circ} \mathrm{C}$ until further processing. In MC patients and controls, they were taken from the right flexure, whereas in UC patients they were taken from macroscopically affected areas of the colon. The colonoscopies were performed at the division of Gastroenterology, Örebro University Hospital, Sweden, between March 2009 and October 2011. All patients in this study had provided written informed consent. The study was approved by the regional ethical committee of Örebro Uppsala County, Sweden (ID number 2008/278; 081015).

\subsection{Total DNA/RNA Extraction, cDNA Synthesis, and Relative} Quantification by Real-Time PCR. Total DNA and RNA were extracted from the same biopsy with AllPrep DNA/RNA Mini Kit (Qiagen, Hilden, Germany) according to the manufacturer's protocol. One $\mu \mathrm{g}$ of total RNA was used for firststrand cDNA synthesis with random primers, using a highcapacity cDNA reverse transcriptase kit, according to the manufacturer's instructions (Applied Biosystems, Foster City, CA, USA). Both DNA and CDNA were stored at $-80^{\circ} \mathrm{C}$ until further processing.

Real time PCR was performed using the thermal cycler TaqMan 7900 Fast Real-Time PCR System (Applied Biosystems) with 7900 Fast Sequence Detection and Relative Quantification software packages. Combined primers and probes for CD3 $\gamma$, GAPDH, and TRECs were purchased from Applied Biosystems: CD3G: Hs00962186_ml and GAPDH: Hs99999905_ml. The sequences for TRECs primers and probes were as follows: HuTRECs Forward; $5^{\prime}$-CCA TGC TGA CAC CTC TGG TT-3', HuTRECs Reverse; $5^{\prime}$-TCG TGA GAA CGG TGA ATG AAG- $3^{\prime}$; and HuTRECs Probe: FAM-5'-CAC GGT GAT GCA TAG GC ACCT GC-3'TAMRA. PCR reactions were performed in $15 \mu \mathrm{L}$ volume using 1X TaqMan Universal PCR Master Mix (Applied 


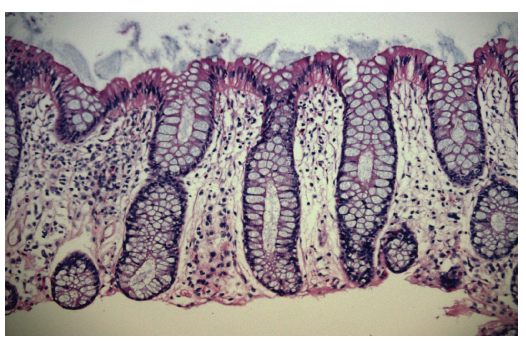

(a)

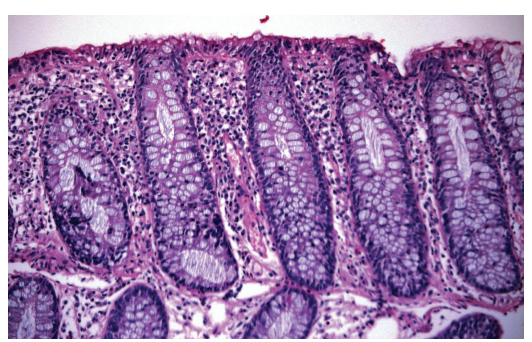

(b)

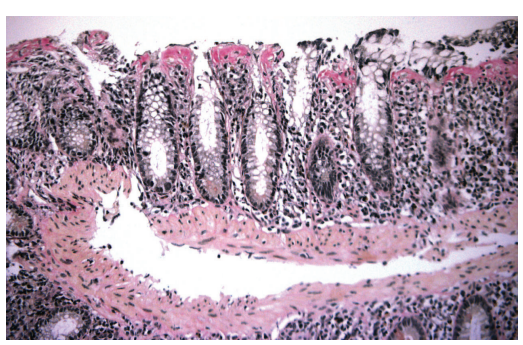

(c)

FIGURE 1: Human colonic biopsies showing (a) normal colonic mucosa; (b) typical findings of lymphocytic colitis, including epithelial cell damage with increased numbers of IELs, and infiltration of lymphocytes in the lamina propria; (c) typical findings of collagenous colitis, including increased thickness of the subepithelial collagen layer, inflammation of lamina propria, and epithelial cell damage with IELs. Photo: Sune Eriksson Department of Pathology, Örebro University Hospital. ((a) and (b)) H\&E Staining, (c) Van Gieson Staining.

TABLE 1: Demographic features of the patients and controls.

\begin{tabular}{|c|c|c|c|c|c|c|c|}
\hline & \multicolumn{3}{|c|}{ Age (years) $)^{\mathrm{a}}$ at the time } & \multirow{2}{*}{$\begin{array}{c}\text { Disease duration } \\
\text { (years) }^{\mathrm{a}}\end{array}$} & \multirow{2}{*}{ Bowel movements/day ${ }^{\mathrm{a}}$} & \multicolumn{2}{|c|}{ On treatment (total) } \\
\hline & $\operatorname{Sex}(F / M)$ & of disease diagnosis & of investigation & & & NSAIDs & PPIs \\
\hline $\mathrm{CC}$ & $7 / 1$ & $61(46-81)$ & $63(56-84)$ & $4(0-17)$ & $5(3-12)(n=7) / \mathrm{ND}(n=1)$ & $2(8)$ & $0(8)$ \\
\hline LC & $5 / 0$ & $69(60-86)$ & $69(60-86)$ & $0(0-7)$ & $6(6-20)(n=4) / \mathrm{ND}(n=1)$ & $0(5)$ & $0(5)$ \\
\hline CC-HR & $3 / 0$ & $49(42-59)$ & $58(43-63)$ & $4(1-9)$ & $4(2-4)$ & $0(3)$ & $0(3)$ \\
\hline LC-HR & $6 / 0$ & $64(23-79)$ & $66(23-79)$ & $1(0-3)$ & $4(3-6)(n=5) / \mathrm{ND}(n=1)$ & $0(6)$ & $0(6)$ \\
\hline NI diarrhoea & $13 / 4$ & NA & $58(22-77)$ & & $4(n=1) / \mathrm{ND}(n=16)$ & ND & ND \\
\hline UC & $2 / 2$ & NA & $54(33-67)$ & & $10(n=1) / \mathrm{ND}(n=3)$ & ND & ND \\
\hline Controls & $6 / 4$ & NA & $64(42-88)$ & & & ND & ND \\
\hline
\end{tabular}

CC: collagenous colitis; LC: lymphocytic colitis; CC-HR: CC-histopathological remission; LC-HR: LC-histopathological remission; NI diarrhoea patients: noninflamed diarrhoea patients; NSAID: nonsteroidal anti-inflammatory drugs, and PPIs: proton pump inhibitors.

${ }^{a}$ Median (min-max), ND: no data, NA: not applicable.

Biosystems) and $100 \mathrm{ng}$ cDNA for CD3 $\gamma$ and $100 \mathrm{ng}$ DNA templates for TRECs analysis. For TRECs analysis, $15 \mu \mathrm{L}$ PCR reactions contained $11 \mu \mathrm{M}$ of each primer, $2.5 \mu \mathrm{M}$ of probe, $7.5 \mu \mathrm{L}$ of TaqMan Universal PCR Master Mix, and nuclease free water. PCR cycling conditions were as follows: Step one: $95^{\circ} \mathrm{C}$ for $2 \mathrm{~min}$, step two: $95^{\circ} \mathrm{C}$ for $3 \mathrm{~s}$ and $60^{\circ} \mathrm{C}$ for $30 \mathrm{~s}$. The second step was repeated 45 times. Reactions were run in triplicates in a 96-well plate. Both DNA and mRNA were prepared from the same tissue and all the samples for CD3 $\gamma$ and TRECs assays were analyzed in the same plate to exclude assay to assay variability.

The relative values were calculated by the $2^{(-\Delta \Delta \mathrm{Ct})}$ method, where TREC expression levels were normalized against CD3 $\gamma$ mRNA, which was used as a reference for the total amount of T cells. CD3 $\gamma$ expression levels were normalized against the reference gene GAPDH and expressed as relative expression.

2.3. Statistical Analysis. Mann-Whitney two-tailed nonparametrical test was used for statistical comparison between groups, and data are presented with median values in the graphs. Differences were considered statistically significant when $P \leq 0.05$. One patient in LC-HR group was defined as a statistical outlier $(P<0.05)$ according to Grubbs test, and therefore data from this patient was excluded while performing statistical comparison between groups. Spearman correlation analysis was performed to correlate TREC levels to age. Statistical analyses were performed using the GraphPad Prism 5 software for Windows.

\section{Results}

3.1. Reduced TREC Levels in the Mucosa of CC and LC Patients. We first investigated the TREC levels in the colonic biopsies relative to the amount of $\mathrm{CD}^{+} \mathrm{T}$ cells. The median TREC levels were lower in CC and LC as well as in LC-HR patients compared to controls (Figure 2). In fact, four out of eight of the CC patients, two out of five of the LC patients, and two out of six of the LC-HR patients (mean 42\%) had undetectable level of TRECs compared to three out of ten in the control group (30\%). However, the changes did not reach statistical significance. In contrast, CC patients in histological remission (CC-HR) did not have altered median TREC levels compared to controls despite clinical symptoms of active CC. Of the two CC patients who were treated with NSAIDs, one presented with the highest TREC levels within the group whereas the other patient had undetectable levels of TRECs, suggesting that NSAIDs did not affect the TREC levels in our study.

In contrast to the $\mathrm{MC}$ patients, but similar to our previous studies on isolated mucosal lymphocytes [17], increased levels of TREC were observed in UC patients, median (range) 0.6 (0.0-1.2) compared to controls. The noninflamed diarrhoea patients presented with very close to statistically significantly 


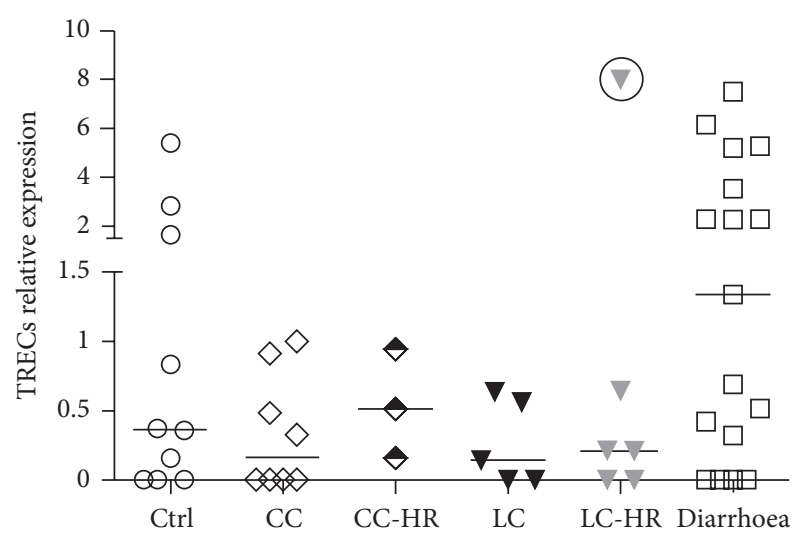

FIgURE 2: Levels of $\mathrm{T}$ cell receptor excision circles (TRECs) in colonic mucosa of patients with collagenous colitis (CC), CChistopathological remission (CC-HR), lymphocytic colitis (LC), LC-histopathological remission (LC-HR), noninflamed diarrhoea patients and controls. The relative expression was calculated by the $2^{(-\Delta \Delta \mathrm{Ct})}$ method. Each symbol represents data from one individual. One symbol with the circle in LC-HR group represents a statistical outlier, and was excluded from the statistical calculations. Controls, $n=10$; CC, $n=8$; CC-HR, $n=3$; LC, $n=5$; LC-HR, $n=6$; and noninflamed diarrhoea, $n=17$.

enhanced TRECs level $(P=0.054)$ compared to controls. No differences could be detected in median TREC levels in either CC-HR or LC-HR patients compared to active CC and LC patients.

Thymus size and activity decrease with increasing age. We therefore investigated the possible correlation between age and TRECs levels in the control group, where the age span was wider. However, we found no significant correlation $(P=0.32)$ between reduced TREC levels and increasing age (data not shown).

3.2. Upregulated CD3 $\gamma$ mRNA Levels in LC Patients. We also recorded the total amount of $\mathrm{CD}^{+} \mathrm{T}$ lymphocytes in the colonic biopsies, by estimating the mucosal mRNA levels for the $\mathrm{CD} 3 \gamma$ gene. Although there was a trend towards increased relative expression of $\mathrm{CD} 3$ in all MC subgroups examined, the upregulation reached statistical significance only in LC patients $(P<0.05)$ compared to controls (Figure 3$)$. LC-HR patients presented with reduced expression of $\mathrm{CD} 3$ gamma compared to LC patients with histologically active disease (Figure 3).

In fact, LC patients had significantly increased CD3 gamma mRNA levels also compared to CC $(P<0.01)$, CCHR $(P<0.05)$, and noninflamed diarrhoea patients $(P<$ 0.01) (Figure 3 ).

\section{Discussion}

This study investigated TRECs level in the colonic mucosa of patients with CC and LC. Here we demonstrate lower levels of TRECs in the mucosa of both CC and LC patients compared to controls, suggesting that the previously observed increased numbers of T cells in the mucosa of CC and LC patients [16]

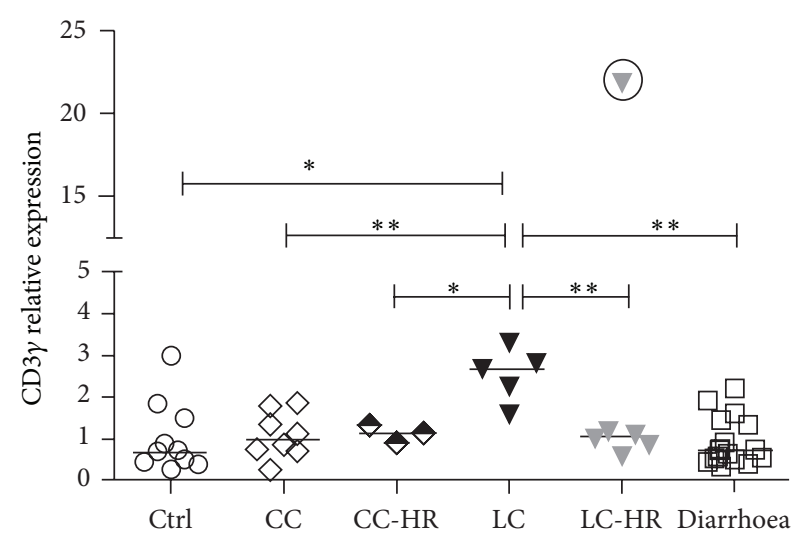

Figure 3: Mucosal mRNA levels of CD3 $\gamma$ in patients with collagenous colitis (CC), CC-histopathological remission (CC-HR), lymphocytic colitis (LC), LC-histopathological remission (LC-HR), noninflamed diarrhoea patients, and controls. The relative expression was calculated by the $2^{(-\Delta \Delta \mathrm{Ct})}$ method. Each symbol represents data from one individual. One symbol with the circle in LC-HR group represents a statistical outlier and was excluded from the statistical calculations. Controls, $n=10$; CC, $n=8$; CC-HR, $n=3$; LC, $n=5$; LC-HR, $n=6$; and noninflamed diarrhoea, $n=17$. LC patients showed statistically significant differences $\left({ }^{*, * *} P<0.05\right.$, $P<0.01)$ compared to other groups.

are due to the expansion of local resident $\mathrm{T}$ cells rather than direct recruitment of recent thymic emigrants to the mucosa. This is in contrast to our previous report on increased levels of TRECs in mucosal lymphocytes from the colonic mucosa of UC patients (but not CD patients) compared to controls [17]. As that study was performed on isolated lymphocytes from resected colons, we also investigated TREC levels in colonic biopsies from 4 UC patients in the present study, and similarly to our previous observations [17] we found increased TREC levels compared to controls.

These results indicate major differences in pathogenesis between UC/CD patients and MC patients, where the latter would likely not benefit from treatment with antibodies blocking homing to the intestinal mucosa, where, for example, Natalizumab has shown good responses in CD patients [18]. This also shows clearly that it is indeed a local mucosal antigen triggering the $\mathrm{T}$ cell activation in MC pathology, possibly by one or several microbiota-derived antigens or by drugs that may aggravate MC in some patients [19].

In the absence of reliable phenotypic markers for RTE, TRECs quantification has become the method of choice to monitor newly generated $\mathrm{T}$ cells migrating to the periphery [9] and has been used to determine thymic export in patients with rheumatoid arthritis [20,21], multiple sclerosis [22] and UC [17]. Reduced TREC levels have also been observed in peripheral blood lymphocytes from patients with rheumatoid arthritis [20,21] and multiple sclerosis [22], compared to controls. However, reduced TREC levels in peripheral blood lymphocytes might very well be accompanied by increased levels in the inflamed tissue. In fact, we have previously published data showing a trend towards reduced TREC levels in 
peripheral blood lymphocytes from patients with ulcerative colitis [17].

We recently published an immunohistochemical [23] as well as a flow cytometric study [16] demonstrating elevated proportions of both LPLs and IELs expressing the Ki67 ${ }^{+}$ phenotype, identifying proliferating cells, in both CC and LC patients. In the latter study both $\mathrm{CD}^{+}$and $\mathrm{CD} 4^{+} \mathrm{T}$ cells were found to harbour increased proportions of cells expressing Ki67. These studies together with the reduced TREC levels in the colonic mucosa of CC and LC patients in the present study suggest that MC patients have local expansion of resident $\mathrm{T}$ cells rather than migration of peripheral lymphocytes to the inflamed mucosa. Involvement of luminal agents [4] and microbes in the gut epithelium $[24,25]$ has previously been reported in MC pathology and our present and previous studies indicate that this initiates strong $\mathrm{T}$ cell responses, resulting in expansion of local $\mathrm{T}$ cells rather than recruitment of peripheral lymphocytes to the mucosa. Constant exposure of luminal agents to the activated $\mathrm{T}$ cells could lead to proinflammatory responses resulting in epithelial damage and chronic inflammation in the intestinal mucosa, as observed in MC.

As MC patients are generally of old age, we were concerned that the recorded decreased median TREC levels could be due to the older age in MC patients compared to controls. Our correlation analysis showed no significant correlation between reduced TREC levels and increasing age in the control group, consisting of a wider age span, suggesting that the observed reduced TREC levels in MC patients are indeed due to the disease itself.

Mucosal mRNA expression of the CD3 $\gamma$ gene was increased in CC and especially in LC patients compared to controls. This is in line with recent data on increased $\mathrm{CD}^{+}$ $\mathrm{T}$ cells in both CC and LC patients [16]. Our recent data on increased proportions of $\mathrm{CD}^{+} \mathrm{T}$ cells but unaltered or reduced proportions of $\mathrm{CD}^{+}{ }^{+} \mathrm{T}$ cells in LC patients [16] indicates that significantly increased mucosal mRNA expression of the CD $3 \gamma$ gene in LC patients stems from mucosal $\mathrm{CD} 8^{+} \mathrm{T}$ cells.

LC-HR patients did not demonstrate normalized TREC levels compared to LC patients, and similarly, we found no significant differences, neither in the proportion of mucosal $\mathrm{T}$ cells expressing Ki67 and the active/memory marker CD45RO nor mucosal mRNA and protein expression of effector T cell cytokines compared to LC patients with histopathologically active disease $[16,26]$, suggesting that mucosal $\mathrm{T}$ cell responses in LC-HR patients are as active as in LC patients.

CD $3 \gamma$ mRNA expression was not altered in noninflamed diarrhoea patients, but they presented with dramatically enhanced TREC levels compared to controls, indicating that in contrast to MC patients, the majority of the mucosal T cells of noninflamed diarrhoea patients are recently migrated from the periphery to the intestinal mucosa. This group of patient is likely heterogeneous and the cause(s) of their symptoms is unknown. The most plausible cause of diarrhoea in these patients is diarrhoea predominant irritable bowel syndrome (IBS-D). Several studies have reported low-grade mucosal inflammation and immune activation in IBS patients [27-29], including increased numbers of $\mathrm{CD}^{+}$LPLs [29], but TREC levels in IBS patients have not been assessed previously. Our recent flow cytometry data showing increased numbers of $\mathrm{CD}^{+}$LPLs in the mucosa of noninflamed diarrhoea patients, albeit still lower than in MC patients [16], is similar to the immunohistochemical findings in IBS patients by Cremon et al. [28]. Together this suggests that noninflamed diarrhoea patients represent patients with IBS-D.

Our study also indicates that the concentration of TRECs in mucosal biopsies could potentially be of future clinical value. According to our data, a patient with diarrhoea with a relative TREC expression $>1.2$ can be excluded from a microscopic colitis diagnosis (Figure 2).

The observations in the current study should be interpreted with caution, as it is performed on small cohorts.

In conclusion, this study demonstrates lower levels of TRECs in the mucosa of both CC and LC patients compared to controls, suggesting local expansion of resident $\mathrm{T}$ lymphocytes in the inflamed mucosa of MC patients. Future investigations would try to sort $\mathrm{CD}^{+}$intraepithelial and lamina propria $\mathrm{T}$ cells, analyze for TREC levels, and perform multivariate analysis by considering different parameters that directly affect the TRECs measurement (age, intracellular degradation of TRECs, cell proliferation, etc.).

\section{Authors' Contribution}

Ashok Kumar Kumawat and Kristina Elgbratt participated in the creation of study design and carried out the studies. Ashok Kumar Kumawat performed data analysis and drafted the paper. Johan Bohr and Curt Tysk performed the colonoscopies and biopsy collections and provided all clinical information about the patients. Ashok Kumar Kumawat, Elisabeth Hultgren Hörnquist, Johan Bohr, and Curt Tysk participated in the finalization of the paper. Elisabeth Hultgren Hörnquist was in charge of the creation of study design, coordination and data analysis. All authors read and approved the final paper.

\section{Acknowledgments}

This study was supported by grants from Swedish Society of Medicine (Bengt Ihre Foundation, Grants SLS249571/2012 and SLS-176271/2011) and Örebro University Hospital Research Foundation. The authors are very grateful to the nursing staff and the gastroenterologists at the Division of Gastroenterology, Örebro University Hospital, for their excellent support in collecting the mucosal biopsies. They also thank Dr. Hilja Strid for her technical guidance.

\section{References}

[1] C. Tysk, J. Bohr, N. Nyhlin, A. Wickbom, and S. Eriksson, "Diagnosis and management of microscopic colitis," World Journal of Gastroenterology, vol. 14, no. 48, pp. 7280-7288, 2008.

[2] D. S. Pardi and C. P. Kelly, "Microscopic colitis," Gastroenterology, vol. 140, no. 4, pp. 1155-1165, 2011. 
[3] A. Munch, D. Aust, J. Bohr et al., "Microscopic colitis: current status, present and future challenges: statements of the European Microscopic Colitis Group," Journal of Crohn's and Colitis, vol. 6, no. 9, pp. 932-945, 2012.

[4] G. Jarnerot, C. Tysk, J. Bohr, and S. Eriksson, "Collagenous colitis and fecal stream diversion," Gastroenterology, vol. 109, no. 2, pp. 449-455, 1995.

[5] S. Brand, "Crohn's disease: Thl, Th17 or both? The change of a paradigm: new immunological and genetic insights implicate Th17 cells in the pathogenesis of Crohn's disease," Gut, vol. 58, no. 8, pp. 1152-1167, 2009.

[6] M. Tsuchiya, T. Hibi, M. Watanabe et al., "Thymectomy in ulcerative colitis: a report of cases over a 13 year period," Thymus, vol. 17, no. 2, pp. 67-73, 1991.

[7] M. Fritsch Fredin, K. Elgbratt, D. Svensson, L. Jansson, S. Melgar, and E. Hultgren Hörnquist, "Dextran sulfate sodiuminduced colitis generates a transient thymic involution-impact on thymocyte subsets," Scandinavian Journal of Immunology, vol. 65 , no. 5, pp. 421-429, 2007.

[8] K. Elgbratt, M. Bjursten, R. Willén, P. W. Bland, and E. H. Hörnquist, "Aberrant T-cell ontogeny and defective thymocyte and colonic T-cell chemotactic migration in colitis-prone G $\alpha \mathrm{i} 2-$ deficient mice," Immunology, vol. 122, no. 2, pp. 199-209, 2007.

[9] R. Somech, "T-cell receptor excision circles in primary immunodeficiencies and other T-cell immune disorders," Current Opinion in Allergy and Clinical Immunology, vol. 11, no. 6, pp. 517-524, 2011.

[10] M. D. Hazenberg, M. C. Verschuren, D. Hamann, F. Miedema, and J. J. van Dongen, "T cell receptor excision circles as markers for recent thymic emigrants: basic aspects, technical approach, and guidelines for interpretation," Journal of Molecular Medicine, vol. 79, no. 11, pp. 631-640, 2001.

[11] T. M. Breit, M. C. M. Verschuren, I. L. M. Wolvers-Tettero, E. J. van Gastel-Mol, K. Hählen, and J. J. M. van Dongen, "Human T cell leukemias with continuous $\mathrm{V}(\mathrm{D}) \mathrm{J}$ recombinase activity for TCR- $\delta$ gene deletion," Journal of Immunology, vol. 159, no. 9, pp. 4341-4349, 1997.

[12] F. Livak and D. G. Schatz, "T-cell receptor $\alpha$ locus V(D)J recombination by-products are abundant in thymocytes and mature T cells," Molecular and Cellular Biology, vol. 16, no. 2, pp. 609-618, 1996.

[13] A. Fischer, S. Hacein-Bey-Abina, and M. Cavazzana-Calvo, "20 years of gene therapy for SCID," Nature Immunology, vol. 11, no. 6, pp. 457-460, 2010.

[14] L. Yin, C. A. Rodriguez, W. Hou et al., "Antiretroviral therapy corrects HIV-1-induced expansion of $\mathrm{CD}^{+} \mathrm{CD} 45 \mathrm{RA}^{+} \mathrm{CD} 27-$ CD1labright activated T cells," Journal of Allergy and Clinical Immunology, vol. 122, no. 1, pp. 166-172, 2008.

[15] P. P. Tagkalidis, P. R. Gibson, and P. S. Bhathal, "Microscopic colitis demonstrates a $\mathrm{T}$ helper cell type 1 mucosal cytokine profile," Journal of Clinical Pathology, vol. 60, no. 4, pp. 382-387, 2007.

[16] A. K. Kumawat, H. Strid, K. Elgbratt, C. Tysk, J. Bohr, and E. Hultgren Hörnquist, "Microscopic colitis patients have increased proportions of $\mathrm{Ki}^{+} 7^{+}$proliferating and $\mathrm{CD} 45 \mathrm{RO}^{+}$ active/memory $\mathrm{CD} 8^{+}$and $\mathrm{CD}^{+} 8^{+}$mucosal T cells," Journal of Crohn's and Colitis, 2012.

[17] K. Elgbratt, G. Kurlberg, M. Hahn-Zohric, and E. Hultgren Hörnquist, "Rapid migration of thymic emigrants to the colonic mucosa in ulcerative colitis patients," Clinical and Experimental Immunology, vol. 162, no. 2, pp. 325-336, 2010.
[18] S. R. Targan, B. G. Feagan, R. N. Fedorak et al., "Natalizumab for the treatment of active Crohn's disease: results of the ENCORE trial," Gastroenterology, vol. 132, no. 5, pp. 1672-1683, 2007.

[19] L. Beaugerie and D. S. Pardi, "Review article: drug-induced microscopic colitis-proposal for a scoring system and review of the literature," Alimentary Pharmacology and Therapeutics, vol. 22, no. 4, pp. 277-284, 2005.

[20] K. Koetz, E. Bryl, K. Spickschen, W. M. O’Fallon, J. J. Goronzy, and C. M. Weyand, "T cell homeostasis in patients with rheumatoid arthritis," Proceedings of the National Academy of Sciences of the United States of America, vol. 97, no. 16, pp. 92039208, 2000.

[21] F. Ponchel, A. W. Morgan, S. J. Bingham et al., "Dysregulated lymphocyte proliferation and differentiation in patients with rheumatoid arthritis," Blood, vol. 100, no. 13, pp. 4550-4556, 2002.

[22] A. Hug, M. Korporal, I. Schröder et al., “Thymic export function and $\mathrm{T}$ cell homeostasis in patients with relapsing remitting multiple sclerosis," Journal of Immunology, vol. 171, no. 1, pp. 432-437, 2003.

[23] C. Göranzon, A. K. Kumawat, E. Hultgren-Hörnqvist et al., "Immunohistochemical characterization of lymphocytes in microscopic colitis," Journal of Crohn's and Colitis, 2013.

[24] A. Münch, J. D. Söderholm, Å. Öst, and M. Ström, "Increased transmucosal uptake of E. coli K12 in collagenous colitis persists after budesonide treatment," American Journal of Gastroenterology, vol. 104, no. 3, pp. 679-685, 2009.

[25] J. Bohr, R. Nordfelth, G. Järnerot, and C. Tysk, "Yersinia species in collagenous colitis: a serologic study," Scandinavian Journal of Gastroenterology, vol. 37, no. 6, pp. 711-714, 2002.

[26] A. K. Kumawat, H. Strid, C. Tysk, J. Bohr, and E. H. Hornquist, "Microscopic colitis patients demonstrate a mixed Th17/Tc17 and Th1/Tc1 mucosal cytokine profile," Molecular Immunology, vol. 55, no. 3-4, pp. 355-364, 2013.

[27] C. Martinez, A. Gonzalez-Castro, M. Vicario, and J. Santos, "Cellular and molecular basis of intestinal barrier dysfunction in the irritable bowel syndrome," Gut Liver, vol. 6, no. 3, pp. 305315,2012

[28] C. Cremon, L. Gargano, A. M. Morselli-Labate et al., "Mucosal immune activation in irritable bowel syndrome: genderdependence and association with digestive symptoms," American Journal of Gastroenterology, vol. 104, no. 2, pp. 392-400, 2009.

[29] V. S. Chadwick, W. Chen, D. Shu et al., "Activation of the mucosal immune system in irritable bowel syndrome," Gastroenterology, vol. 122, no. 7, pp. 1778-1783, 2002. 


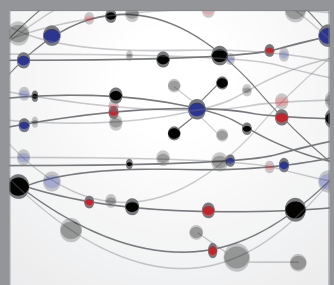

The Scientific World Journal
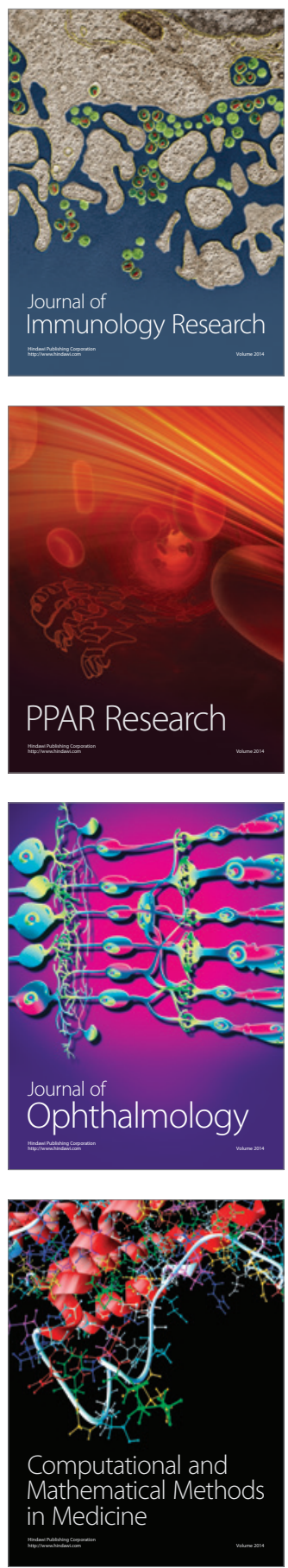

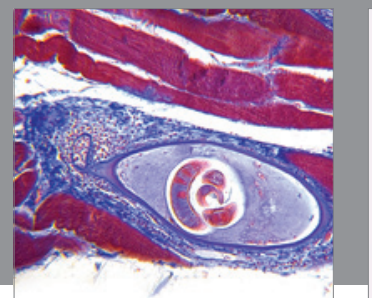

Gastroenterology

Research and Practice
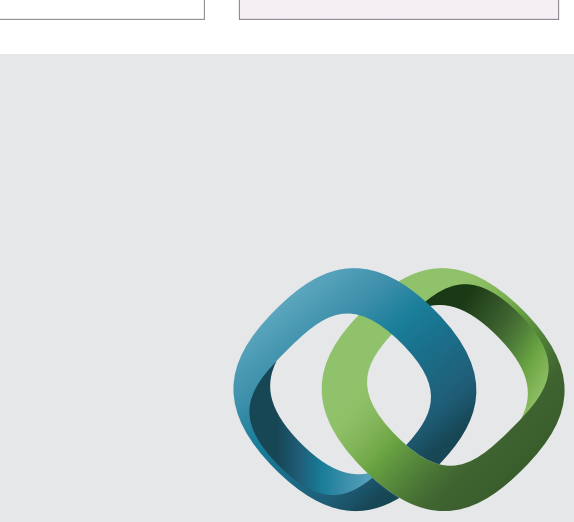

\section{Hindawi}

Submit your manuscripts at

http://www.hindawi.com
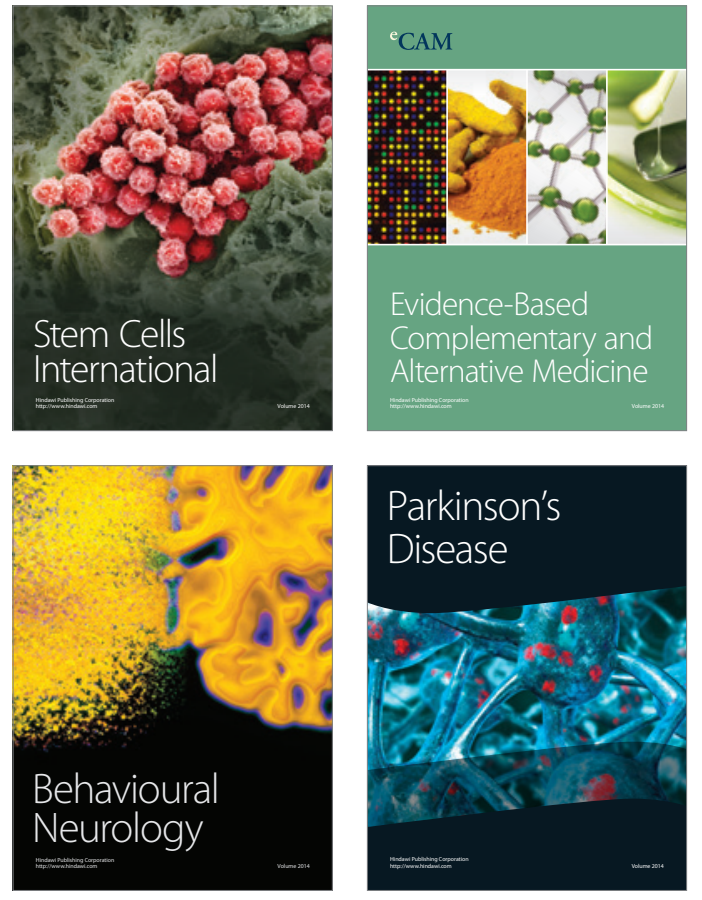
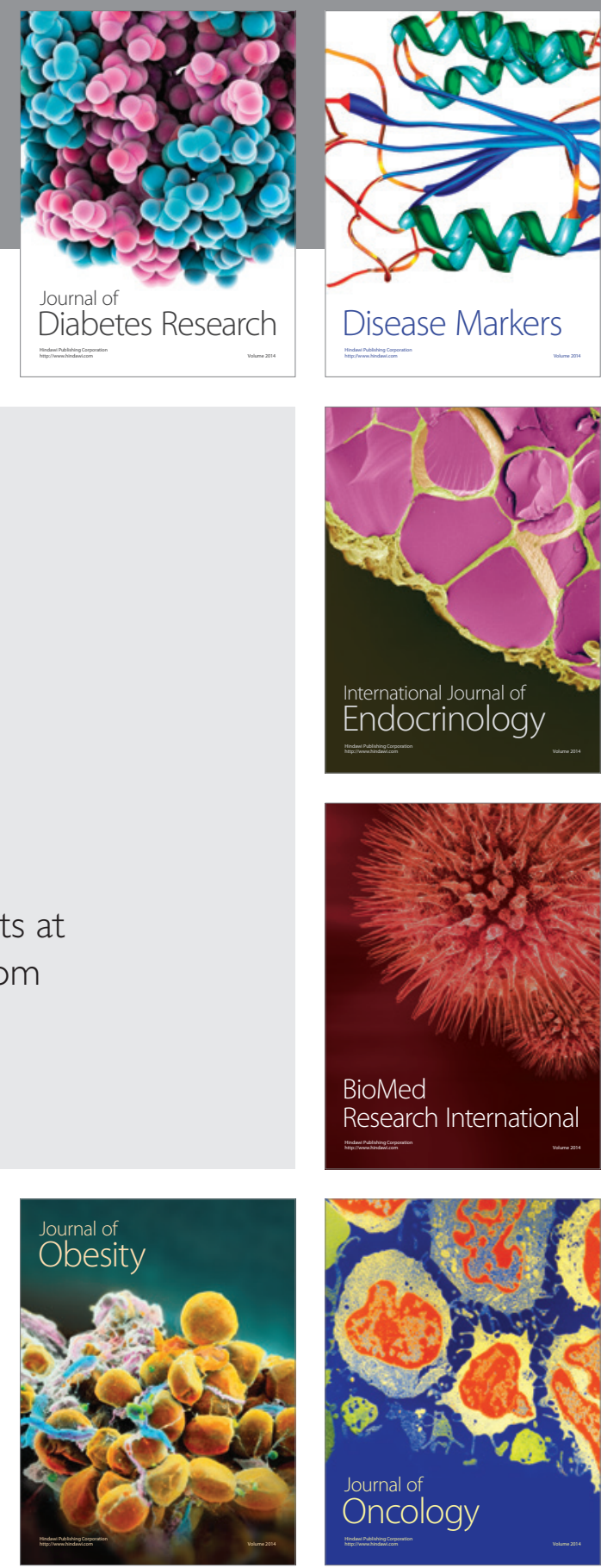

Disease Markers
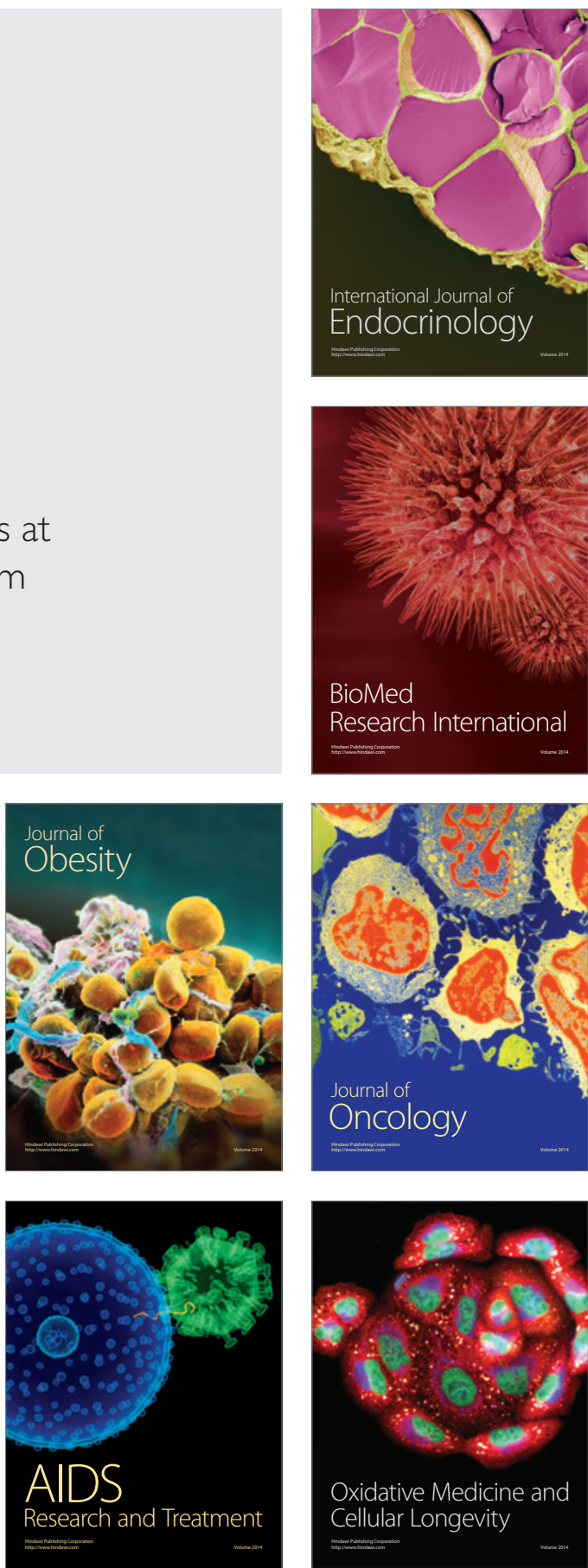